\title{
Non-deictic Tenses in Conditionals
}

\author{
Katrin Schulz \\ Universiteit van Amsterdam
}

\section{Introduction}

This paper is about the interpretation of tenses in conditional sentences. It focuses on a particular cluster of data centering around the observation that tenses in the consequent of a conditional can obtain a non-deictic interpretation. As we will see, there is no need for something very extravagant or new to deal with these observations. We can do with the standard analysis of indicative conditionals using quantification over possible worlds (Kratzer 1979, 1981): a conditional If $A$, then $C$ is true with respect to a set of possible worlds $B$ if $C$ is true at all, most or few worlds in $B$ at which $A$ is true. This standard analysis will be combined with a similarly well-known approach towards the meaning of the English tenses: a nondeictic, relative semantics, as, for instance, proposed in Abusch (1997) and Stechow (2005). The original contribution of this paper lies primarily in how it spells out the model theory for this standard semantics, particularly in the way it models the asymmetry between closed past and open future. In contrast to the standard position based on work of Kamp (1978), the present paper formalizes the openness of the future by leaving possible worlds generally undefined for the future. This shift in the conception of the future will have consequences for the temporal properties of modal bases $B$ that enter the semantics of modal/conditional constructions. As will be shown below, this affects the interpretation of tenses in modal/conditional constructions. In particular, it will explain the cluster of observations that the paper intends to account for.

There are many questions concerning the interpretation of conditional sentences that this paper is silent on (for instance, the interpretation of aspect in conditional constructions, presupposition projection and the issue of how to model counterfactual reasoning). The decision to leave these topics out results from the conviction that these issues are independent from the specific problem the paper does address. This is also the reason for why the present proposal is worked out within a very simple semantic framework. This will enable the reader to easily fit in his/her favored approach to deal with the issues of modal/conditional semantics that (s)he is working on.

Thanks to the audience at SALT XVIII in Amherst, at JSM 2008 in Toulouse and the LeGO community in Amsterdam for comments on the ideas presented here. In particular, I'd like to thank Kay von Fintel, Michael Franke, Kyle Rawlins, and Roger Schwarzschild. 


\subsection{A basic theory for the semantics of the English tenses}

As said above, this paper addresses certain problematic observations concerning the interpretation of tense in conditional sentences. But before we can apprehend that some observation is problematic we first need to formulate a basic theory that fixes our expectations for how tenses in conditionals are interpreted. Using Ockham's Razor, we assume first that the semantics of the English tenses in conditionals is the same as in other sentences. Furthermore, we assume a very simple and basic semantics for the tenses. ${ }^{1}$ Intuitively, tenses locate the time at which the eventuality described in their scope is situated relative to some other time. Let us introduce some useful terminology and call the first time parameter the denotation time of a tense and the time with respect to which the denotation time is located the anchor of a tense. The basic theory of tense that we start with embraces the following three claims, which are summarized in the interpretation rules for the tenses stated in definition $1 .^{2}$

- The tenses are deictic: the anchor is the utterance time.

- The tenses are anaphoric: the denotation time is contextually given.

- The Simple Past demands that the denotation time lies before the anchor time and the Simple Present that the denotation time is the anchor time. ${ }^{3}$

Definition 1 A simple deictic semantics for the tenses

$$
\begin{aligned}
& \text { Past }_{1}: \lambda P \lambda t_{0} \lambda w \cdot t_{1}<t_{0} \& P(w)\left(t_{1}\right) \\
& \text { Pres }_{2}: \lambda P \lambda t_{0} \lambda w . t_{2}=t_{0} \& P(w)\left(t_{2}\right)
\end{aligned}
$$

The rest of the paper is structured as follows. In the next section the target observations the paper intends to account for will be introduced. Section three develops the theory with which we want to address the observations. In section four it will be shown in detail how the theory can account for each of the observations made in section two. This section is then followed by a discussion of some implications of the proposal made. Section five sums up and concludes the paper.

\section{Tense in conditionals: the data}

Equipped with the basic theory for the interpretation of the tenses stated above, let us now have a look at the interpretation of tenses in conditional contexts. We start with the antecedent. A first observation that catches the eyes is that in the antecedent

\footnotetext{
${ }^{1}$ This is the core of the analysis that can be found in many modern semantic textbooks, for instance, Kamp and Reyle (1993).

${ }^{2} t_{0}$ is a special variable reserved for reference to the utterance time.

${ }^{3}$ These inferences are generally taken to be presuppositions of the tenses. I agree with this analysis, but for the purpose of the paper it is not necessary to distinguish presuppositions and therefore this information is placed in the asserted content of the tenses.
} 
of indicative conditionals the Simple Present can select a future denotation time to the future. Compare, for instance, (1) which is a standard example of an indicative conditional.

(1) If I win the competition (tomorrow), I will buy an expensive car.

The basic theory of tenses we started with cannot account for this use of the Simple Present, because it does not allow the Simple Present to select a future denotation time. This problem is not bound to conditional contexts. Also in simple sentences the Simple Present can chose a future denotation time (see 2a). But there is something peculiar about the conditional context. As it has often be observed (see Kaufmann (2005) and the references mentioned there), a futurative use of the Simple Present in simple sentences is heavily restricted. We cannot say (2b).

a. The train leaves at 10 p.m. tomorrow.

b. I win the competition (tomorrow).

Intuitively, we can only use the Simple Present in simple sentences with a future denotation time, if the future fact described is perceived as predetermined at the utterance time (for an extensive discussion, see Kaufmann (2005)). This restriction, however, does not extend to the use of the Simple Present in the antecedent of indicative conditionals. Neither does (1) presuppose that it is predetermined whether I win the competition, nor is it understood as claiming that if it is predetermined that I win the competition, I will buy an expensive car. To summarize, the following observations have to be explained.

Observation 1. In the antecedent of an indicative conditional that refers to the future the Simple Present is used.

Observation 2. In contrast to simple sentences, the use of the Simple Present in the antecedent of an indicative conditional about the future does not imply predetermination of the described eventuality in the future.

After this discussion of the antecedent, we now turn to the consequent of English conditional sentences. Let's have a look, for instance, at example (3). This sentence has a reading according to which the interview mentioned takes place before John leaves smiling, but still in the future of the utterance time. Thus, apparently, the Simple Past in the consequent is not anchored in the utterance time, but in the denotation time of the tense of the antecedent: the time of John's leaving. ${ }^{4}$

(3) If John comes out smiling, the interview went well.

\footnotetext{
${ }^{4}$ Notice that this is not a sequence of tense phenomenon. The Simple Past in the consequent is not semantically void, but still expresses pastness.
} 
The observation extends to the Simple Present. In its generic reading, example (4a) cannot have an interpretation according to which the rising of the temperature takes place before the bending of the strip. One might argue that the alternative reading is excluded by world knowledge. But this reading is admissible if we express the same conditional relation as fact about the past (4b).

(4) a. If the strip bends, the temperature rises.

b. If the strip bent, the temperature rose.

So far, we have only discussed examples without modal verb in the consequent. But the observation can be made for explicitly modalized conditionals as well. This is somewhat less easy to observe, because, as discussed in more details below, the shift of the anchor to the denotation time in the antecedent is not obligatory. It is only obligatory given a particular reading of the conditional, or to use the terminology of Kratzer $(1979,1981)$, given the choice of the modal base. Consider, for instance, the conditionals in (5), reading the dependency between antecedent and consequent as causal dependence (he passed the examen because he practiced the night before). With this reading variant (5a), without perfect, is marked and (5b) should be used. The use of the perfect is necessary because the anchor for the temporal interpretation of the modal phrase is shifted to the future denotation time of the Simple Present in the antecedent.

(5) a. If tomorrow he passes the examen, he will practice all night.

b. If tomorrow he passes the examen, he will have practiced all night.

The same optional shift of the anchor can be observed with subjunctive conditionals. ${ }^{5}$ Translating the indicative example (3) into the subjunctive, the perfectfree variant (6a) is out and (6b) has to be used. Again, the fact that you have to use the perfect shows that the evaluation time of the modal is shifted to the future denotation time of the Simple Present in the antecedent. The perfect is necessary to access a time in the future of the utterance time and in the past of the denotation time of the tense in the antecedent.

(6) a. ??If John came out smiling, the interview would go well.

b. If John came out smiling, the interview would have gone well.

We summarize our observations.

Observation 3. In conditionals the anchor of the tense in the consequent can be shifted to the denotation time of the tense in the antecedent.

There are two related observations concerning the shift of the anchor in the consequent. First, the shift of the anchor only occurs if the tense in the antecedent indeed refers to a future time. We do not observe it, if the antecedent refers to some

\footnotetext{
${ }^{5}$ To my knowledge this has not been observed before.
} 
past time or the utterance time. Second, as already pointed out above, the future shift of the anchor is not obligatory, even in case the antecedent does refer to the future. Crouch (1993) additionally observes that in this case the antecedent of the conditional is presupposed to be predetermined at the utterance time. To illustrate his point he presents (7). ${ }^{6}$

(7) If I go to the party, I will have a shower.

It is not entirely clear whether Crouch (1993)'s observation is correct. One of the reviewers suggested (8) as counterexample to Crouch's observation. This example is fine under a reading that does not presuppose that the antecedent is predetermined at the utterance time. However, it is not obvious that the consequent is a straightforward claim about the utterance time and not about a time interval that includes the utterance time as well as the future denotation time of the antecedent (double access reading). More empirical research is needed.

(8) If the boss comes out of the interview smiling, Mandy is wearing her new perfume.

We summarize the last two observations.

Observation 4. The shift of the anchor in the consequent of conditionals is only observed, if the denotation time in the antecedent is a future time.

Observation 5. The shift of the anchor is not obligatory, even if the tense in the antecedent selects a future denotation time.

\section{The theory}

In this section we will develop an explanation for the observation made above. As said before, we do not need something very extraordinary to this purpose. We will adopt the standard approach towards the interpretation of conditional sentences and combine it with a similarly well-known semantics for the English tenses. The innovative part lies in the way the future is formalized. This alternative formalization is based on a non-standard modeling of the openness of the future. Before we come to this part of the proposal we will first fix interpretation rules for indicative conditionals and the tenses, building on Kratzer (1979, 1981) and Abusch (1997).

\subsection{An interpretation rule for conditionals}

The theory of conditionals adopted here is the standard analysis spelled out formally in terms of quantification over possible worlds: a conditional If $A$, then $C$ is

\footnotetext{
${ }^{6}$ Example (59) on p. 45 of Crouch (1993).
} 
true w.r.t a set of possible worlds $B$ if $C$ is true at all, most or few worlds in $B$ at which $A$ is true. We adopt in particular the well-known implementation of this idea in Kratzer $(1979,1981)$ according to which the quantification is expressed by an (implicit) modal in the logical form of the conditional. In this case $B$ is the contextually given modal base, that enters the interpretation as argument of the modal. A second contextually given argument is an ordering that represents preferences on the elements of the modal base. ${ }^{7}$ Kratzer $(1979,1981)$ 's approach is summarized in figure 1 and the interpretation rule given in definition 2. The formula in definition 2 says that for the conditional to be true it has to be the case that those worlds in the modal base that make the antecedent true and are minimal with respect to the ordering also make the consequent true. There are more sophisticated approaches towards the meaning of indicative conditionals, but most of them share the basic ideas with this well-known and simple approach and for our purposes it is sufficient. The reader is then invited to extend the present proposal to the his/her favored conditional semantics.

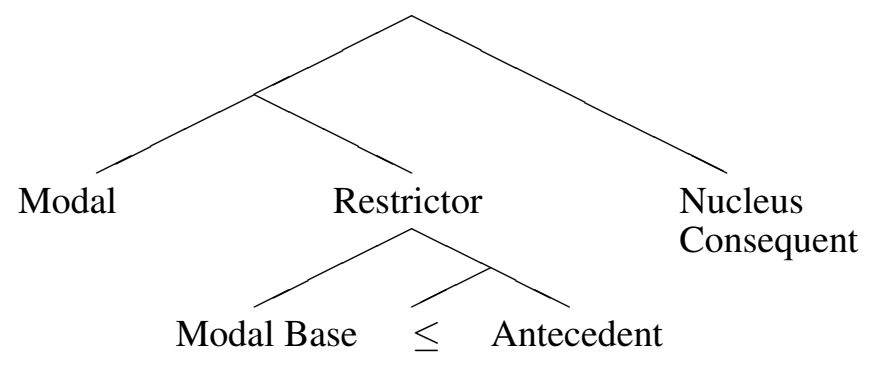

Figure 1: The syntactic structure of conditional sentences

Definition 2 Kratzer's interpretation rule for conditionals ${ }^{8}$

IF $\phi, \psi: \operatorname{MUST}_{M B, \leq}(\phi, \psi)$

$: \lambda w . \forall w^{\prime}: w^{\prime} \in \operatorname{Min}_{M B, \leq}(w, \phi) \rightarrow \psi\left(w^{\prime}\right)$.

Definition 3 The operation $\mathrm{Min}^{9}$

For a set of objects $S$ with $s \in S$ and $S^{*} \subseteq S$, a binary accessibility relation $R$ on $S$ and a partial order $\leq$ on $S$ we define the operation Min as follows:

$\operatorname{Min}_{R, \leq}\left(s, S^{*}\right)=\left\{s^{\prime} \in R(s) \mid s^{\prime} \in S^{*} \& \neg \exists s^{\prime \prime} \in R(s): s^{\prime \prime} \in S^{*} \& s^{\prime \prime}<s^{\prime}\right\}$.

\subsection{The interpretation of the tenses}

We started this paper with a simple theory for how to interpret the English tenses. According to this theory the Simple Past expresses that the denotation time of the

\footnotetext{
${ }^{7}$ This paper focuses on indicative conditionals, although with the right semantic theory for subjunctive conditionals in place the proposal should easily extend to them as well.

${ }^{8}$ The modal can differ in different conditionals. All-quantifying MUST is chosen by default.

${ }^{9}$ For the sake of simplicity we adopt in this paper the limit assumption, i.e. we assume that minima always exist.
} 
tense lies before its anchor time and the Simple Present expresses that the denotation time is the anchor time. Furthermore, we assumed that the tenses are anaphoric and deictic, in particular, we assumed that the anchor time is the utterance time. In (4) the interpretation rules for the tenses from section 1.1 are repeated.

Definition 4 A simple deictic semantics for tenses

$$
\begin{aligned}
& \text { Past }_{1}: \lambda P \lambda t_{0} \lambda w . t_{1}<t_{0} \& P(w)\left(t_{1}\right) \\
& \text { Pres }_{2}: \lambda P \lambda t_{0} \lambda w . t_{2}=t_{0} \& P(w)\left(t_{2}\right)
\end{aligned}
$$

This simple theory will now be changed in two respects. For one thing, we will change the interpretation of the Simple Present. Second, we will give up the assumption that tenses are in a strict sense deictic.

\subsubsection{Simple Present as non-pastness}

In section 2 we have already encountered data that made the assumed meaning for the Simple Present dubious. The Simple Present can chose future denotation times. This does not only hold in conditional or modal sentences, but also for simple sentences like (2a), here repeated as (9).

(9) The train leaves at 10 p.m. tomorrow.

We will therefore follow Quirk et al. (1985) and propose that the simple present in English expresses non-pastness instead of just reference to the utterance time.

Definition 5 Preliminary semantics for the Simple Present Pres $_{2}: \lambda P \lambda t_{0} \lambda w . t_{2} \geq t_{0} \& P(w)\left(t_{2}\right)$

\subsubsection{Tenses are non deictic}

According to our simple theory of the semantics of the English tenses the tenses are deictic. They localize the denotation time relative to the utterance time. It has been argued at different places that at least in some contexts the English tenses cannot be interpreted as deictic, as anchored in the utterance time (Abusch 1997, Stechow 2005). For illustration we borrow the example(10) from Abusch (1997). If the Simple Past were deictic and localized the denotation time in the past of the utterance time, then (10) should have a reading according to which the occurrence of the Simple Past in the complement clause denotes a time that lies before the utterance time but still in the future of the denotation time of the past tense marked on believe. Such a reading, however, is impossible.

(10) Last Monday John believed ${ }_{1}$ that he was $_{2}$ in Paris on Tuesday.

To account for examples like (10) Abusch (1997) proposes to follow Montague grammar and let intensional operators bind the evaluation time in their scope. They quantify, hence, not just over possible worlds, but over possibilities $p$, tuples 
of a possible world and a time $\langle w, t\rangle$ (Montagovean indices). The time parameter in this tuple expresses the temporal perspective of the possibility, i.e. what in this possibility is considered to be the present. In other words, intensional operators are proposed to operate on centered propositions. The semantics of 'believe' can then be formalized as in (1), where $\mathbf{B}$ provides the doxastic state of agent $x$ in possibility $p$.

$$
\text { believe : } \lambda P \lambda x \lambda p . \forall p^{\prime}: p^{\prime} \in \mathbf{B}(x, p) \rightarrow P\left(p^{\prime}\right)
$$

This idea can naturally be extended to conditional sentences. The only change necessary in the interpretation rule of definition 2 is the exchange of variables for possible worlds by variables for possibilities. The new interpretation rule we obtain is given in definition 6. According to this rule the temporal perspective in conditional contexts is not set by the utterance time of the conditional but by the temporal perspective of the possibilities in the modal base.

Definition 6 The semantics of conditionals

$$
\begin{aligned}
I F \phi, \psi & : \operatorname{MUST}_{M B, \leq}(\phi, \psi) \\
& : \lambda p . \forall p^{\prime}: p^{\prime} \in \operatorname{Min}_{M B, \leq}(p, \phi) \rightarrow \psi\left(p^{\prime}\right)
\end{aligned}
$$

Furthermore, Abusch (1997) proposes that tenses are not in a strict sense deictic, but evaluated relative to the local evaluation time. In the scope of intensional operators the local evaluation time might differ from the utterance time. The interpretation rules for the tenses are consequently changed to what is stated in definition 7.

Definition 7 The meaning of the tenses

$$
\begin{aligned}
& \text { Past }_{1}: \lambda P \lambda\langle w, t\rangle . t_{1}<t \& P\left(\left\langle w, t_{1}\right\rangle\right) \\
& \text { Pres }_{2}: \lambda P \lambda\langle w, t\rangle . t_{2} \geq t \& P\left(\left\langle w, t_{2}\right\rangle\right)
\end{aligned}
$$

This is not a complete representation of Abusch (1997)'s proposal. She develops this relative semantics for the tenses further in order to account for double access readings and sequence of tense (SOT) phenomena. For the purpose of the present paper we can do with the simpler analysis of definition 7 and assume that the ultimate theory needed to account for SOT phenomena and double access readings reduces in the simple cases to what is proposed here.

\subsection{The ontic modal base}

This section contains the central contribution of the present paper. Its basis is a non-standard explanation of the asymmetry of the future and the past. Building on this the paper proposes an alternative formalization of the ontic modal base. This modal base has been called many different names in the literature: circumvential modal base, metaphysical modal base, or aletic modal base. We choose the term ontic, that has been suggested by Veltman (p.c.), because it better conveys the intended meaning. The ontic modal base will play a crucial role in the explanation of the observations this paper targets at. For, as will be shown in the next section, 
the temporal properties of modal constructions depend on the chosen modal base. More in particular, the future shift of the anchor of English tenses described in observation 3 of section 2 results in case an ontic modal base is chosen.

There exists a certain asymmetry between the past and the future. While the past is fixed and unaffected by our actions, the future is open, undecided and can be manipulated. There is a standard way to model this difference in semantics, that builds essentially on Kamp (1978). According to this position the fact that we perceive the future as open is a consequence of our limited capacities to figure out the future. Thus, the asymmetry of past and future is a purely epistemological effect. Building on this, Kamp (1978) assumes that a sentence can be true without being (perceived to be) decided. The alternative position, defended, for instance, by Aristotle in de interpretatione assumes to the contrary that the undecidedness of the future is metaphysical in nature and, hence, that future facts are actually undecided. In consequence, they do not come with a truth value. Formally, the difference between the two positions shows up in the way possible worlds are defined. While the standard position of Kamp (1978) assumes that possible worlds are completely defined truth functions, the Aristotelian position demands that possible worlds are undefined for the future. In this paper we adopt an intermediate position. Following Aristoteles we claim that the future is generally undecided. But there are exceptions. The future can be defined, if this already follows from facts of the past and the present and non-defeasible general laws. ${ }^{10}$ In the following we will not discuss the different philosophical arguments that can be brought forward for one position or the other. Instead of this, we will show how choosing the described intermediate position can help to solve the linguistic questions at stake.

With this conception of the future in mind we now try to formalize the ontic modal base. Intuitively, this modal base captures the alternative ways the world might turn out to be in the future. According to the standard formalization of the openness of the future, ontic alternatives are defined on the level of possible worlds. Let $w$ be such a possible world. Then, it is said that $w^{\prime}$ is among the ontic alternatives of $w$ at time $t\left(w \cong{ }_{t} w^{\prime}\right)$, if $w^{\prime}$ and $w$ agree with respect to the past and the present (that means that they assign the same interpretations to the non-logical vocabulary for all $t^{*} \leq t$ ) and might differ for any time in the future of $t$.

Adopting the intermediate position towards the future described above, the natural formalization of the ontic alternatives looks different. Ontic alternatives are no longer defined on the level of possible worlds but on the level of possibilities, or centered worlds. Recall from section 3.2.2 that a possibility $p$ is a tuple $\langle w, t\rangle$ consisting of a possible world $w$ and a time $t$, representing the temporal perspective of the possibility, i.e. the time that is according to this possibility the present. Given the chosen conception of the future, not any tuple of a possible world and a time count as proper possibility. In order for $p$ to be proper the possible world $w$ has to be completely defined for the past and the present, but generally undefined for the future of $t$. W is only allowed to be defined for future times if this already follows

\footnotetext{
${ }^{10}$ For a proposal of how this can formally be spelled out, see Schulz (2007).
} 
from facts about the past and the present and general non-defeasible laws.

\section{Definition 8 Possibilities}

A tuple $p=\langle w, t\rangle$ where $w$ is a partially defined interpretation function and $t$ a time is a possibility iff ( $i) w$ is completely defined upto $t$, and (ii) for any property $P$ and time $t^{\prime}>t: P\left(\left\langle w, t^{\prime}\right\rangle\right)$ is defined if and only if this follows from the past, the present and non-defeasible general laws.

Based on this characterization of possibilities the ontic alternatives $O(p)$ of some possibility $p=\langle w, t\rangle$ are defined as the set of possibilities $p^{\prime}=\left\langle w^{\prime}, t^{\prime}\right\rangle$ whose temporal perspective $t^{\prime}$ is identical to $t$ or lies in the future of $t$ and whose world parameter $w^{\prime}$ agrees with $w$ for all points at which $w$ is defined. That means that $w$ and $w^{\prime}$ particularly agree for all points in the past and the present, but if $w$ is defined for some future time $t^{*}$ as well, then also $w^{\prime}$ has to be defined for this point and to agree with $w$ in its definition.

\section{Definition 9 The ontic modal base $O$}

The ontic modal base $O(p)$ of possibility $p=\langle w, t\rangle$ is defined to be the set of possibilities $p^{\prime}=\left\langle w^{\prime}, t^{\prime}\right\rangle$ with $(i) t^{\prime} \geq t$, and (ii) $w \subseteq w^{\prime}$.

Finally, we assume that the ontic modal base $O(p)$ comes with a specific ontic preference order $\leq p$. This order prefers possibilities whose temporal perspective comes closest to the temporal perspective of $p .^{11,12}$

Definition 10 The ordering $\leq p$ on $O(p)$

For a possibility $p$ we define the order $\leq p_{p}$ on the ontic modal base $O(p)$ as follows: $\forall\left\langle w_{1}, t_{1}\right\rangle,\left\langle w_{2}, t_{2}\right\rangle \in O(p):\left\langle w_{1}, t_{1}\right\rangle \leq{ }_{p}\left\langle w_{2}, t_{2}\right\rangle$ iff $t_{1} \leq t_{2}$.

Figure 2 illustrates the definition of a possibility $p=\langle w, t\rangle$. The line of the world parameter is complete for all times before the temporal perspective, but has gaps for future times. Figure 3 depicts some ontic alternatives of this possibility. Of course, $p$ itself is among its ontic alternatives. But there are also other possibilities in this set. They continue the line of complete definition of $p$ to some point in the future of $t$. They have to agree with $w$ everywhere where $w$ is defined, but might fill in the gaps of $w$ differently. So, in principle, this theory supports the same picture of a branching future that we are familiar with from the standard approach towards ontic alternatives, but now the different branches are not defined completely. The order that comes with the ontic modal base prefers possibilities that continue least far into the future. Hence, the possibilities sketched in figure 3 are related to each other in the following way: $p<p^{\prime}<p^{\prime \prime}$.

\footnotetext{
${ }^{11}$ Within $O(p)$, this order is centered in $p$.

${ }^{12}$ Intuitively, the order prefers most similar possibilities. The more details of the future become fixed, the more the resulting possibilities differs from the original one.
} 
Figure 2: A picture of an ontic alternative $p=\langle w, t\rangle$

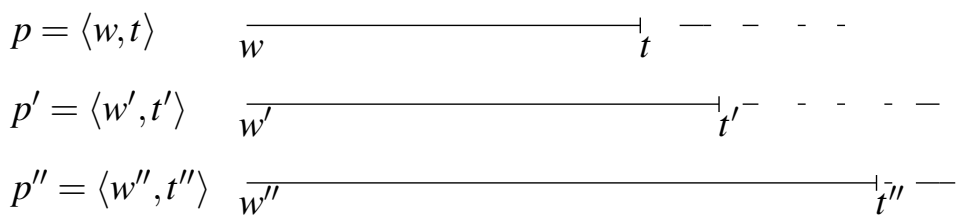

Figure 3: Some ontic alternatives of a possibility $p=\langle w, t\rangle$

\section{Explaining the data}

In this section we will work through the five observations made in section 2 and explain how the present approach can account for them.

\subsection{Observation 1}

The first observation made in section 2 was that in the antecedent of indicative conditionals the Simple Present can take future denotation times. This observations is a problem for approaches that claim that the Simple Present identifies denotation time and anchor. It is not a problem for the approach adopted here that analyses the Simple Present as expressing non-pastness.

\subsection{Observation 2}

The second observation made in section 2 was twofold. First we observed that if in a simple sentence the Simple Present chooses a future denotation time, the described eventuality has to be predetermined at the utterance time. Therefore, the Simple Present can only be used in sentences like (11a) and examples like (11b) are out.

(11) a. The plane arrives at 3 p.m. this afternoon.

b. I win the lottery tomorrow.

The meaning the present theory assigns to sentences like (11b) is given in equation 2. If the time that is assigned to variable $t_{1}$ lies in the future of the local temporal perspective $\left(t_{1}>t\right)$, then the sentence can only be true in possibility $p$, if in $p$ property $P$ is defined to be true at the future time $t_{1}$. However, according to the proposed constraints on what counts as a proper possibility (definition 8 ), this can only be the case if the fact that $P$ is true at $t_{1}$ follows from facts about the present and the past of $p$ together with non-defeasible general laws. This accounts for the observation that in simple sentences the simple present can only be used with 
future reference if the described eventuality is perceived as being predetermined at the utterance time.

$$
\begin{aligned}
(10) & =P R E S_{1}(P) \\
& =\lambda\langle w, t\rangle \cdot \exists t_{1}: t_{1} \geq t \& P\left(\left\langle w, t_{1}\right\rangle\right)
\end{aligned}
$$

The second part of observation 2 was that in the antecedent of conditionals the use of the Simple Present with a future denotation time does not imply predetermination of the described eventuality. In equation 3 the semantics assigned to conditional (1), here repeated as (12), is spelled out. The relevant observation is correctly born out by the present proposal if the possibilities that end up in the restrictor of the quantifier in this formula do not make the antecedent true by predetermination. That means that the antecedent should not be defined to be true at some future time relative to the local temporal perspective of these possibilities.

If I win the competition (tomorrow), I will buy an expensive car.

$$
\begin{aligned}
(12) & =\operatorname{MusT}_{O, \leq}\left(\operatorname{PRES}_{1}(P), \operatorname{PRES}_{2}(Q)\right) \\
& =\lambda p \cdot \forall p^{\prime}: p^{\prime} \in \operatorname{Min}_{O, \leq_{p}}\left(p, \operatorname{PRES}_{1}(P)\right) \rightarrow \operatorname{PRES}_{2}(Q)\left(p^{\prime}\right)
\end{aligned}
$$

Which possibilities will end up in the restrictor set? These are possibilities that (i) are among the ontic alternatives of the evaluation possibility $p$, (ii) make the antecedent $P R E S_{1} P$ true, and (iii) are minimal with respect to the order $\leq p$. Figure 4 illustrates some ontic alternatives for possibility $p$. Let us assume that the truth of $P$ at $t_{1}$ with $t_{1}>t$ is not defined in $p$ itself and also does not become defined in some ontic alternative $p^{*}=\left\langle w^{*}, t^{*}\right\rangle$ of $p$ with $t<t^{*}<t_{1}$. This is what we normally assume for eventualities situated in the future. Then the only possibilities among the ontic alternatives of $p$ in which $P$ is defined and true at $t_{1}$ are either like $p^{\prime}$ whose temporal perspective $t^{\prime}$ is the denotation time $t_{1}$ of the present tense in the antecedent, or possibilities like $p^{\prime \prime}$ that are defined even further into the future, i.e. whose temporal perspective $t_{p^{\prime \prime}}$ is some time in the future of $t_{1}$. However, only possibilities of type $p^{\prime}$ make the antecedent $P R E S_{1} P$ true, because from the local temporal perspective of possibilities like $p^{\prime \prime}$ the fact that $P$ is true at $t_{1}$ is a past fact. Because all possibilities of type $p^{\prime}$ have the same temporal perspective: $t^{\prime \prime}=t_{1}$, the minimization has no effect and the restrictor set contains all of these possibilities. But in these possibilities the antecedent is not predetermined to be true. In $p^{\prime}$ the antecedent of the conditional $P R E S_{1} P$ is a fact about the local present. Hence, the approach correctly predicts that in the antecedent of conditionals the Simple Present can be used without that this implies predetermination of the fact described. ${ }^{13}$

\footnotetext{
${ }^{13}$ If the antecedent is predetermined at the utterance time, or taken to be predetermined at some future time $t^{\prime}$ with $t_{p}<t^{\prime}<t_{1}$, then the restrictor of the modal quantifier will contain those possibilities whose local temporal perspective is the time at which the antecedent becomes predetermined. That this prediction is correct can be illustrated with examples like (1) from Kaufmann (2005) (example (47) in the paper). In this example it is clear that the decision will fall at some future time before the actual future event time. We observe the predicted future-shifted predetermination reading.
} 


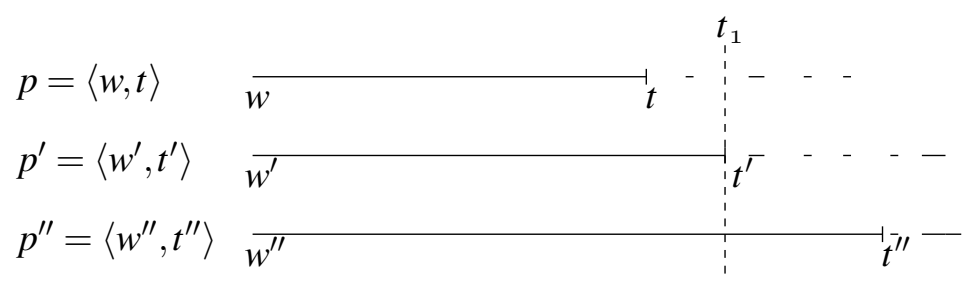

Figure 4: Ontic alternatives for equation 3

\subsection{Observation 3}

Observation 3 concerned the interpretation of tenses in the consequent of conditionals. We observed that the anchor of tenses occurring in the consequent can be shifted to the future. More particularly, the anchor appears to be identified with the denotation time of the tense in the antecedent. This was illustrated with example (3), here repeated as (13), that has a reading according to which the Simple Past in the consequent is anchored at the future denotation time $t_{1}$ of the antecedent and chooses as denotation time $t_{2}$ a time before $t_{1}$ and after the utterance time.

(13) If he comes ${ }_{1}$ out smiling, the interview went ${ }_{2}$ well.

Lets see whether the approach advocated here can account for this observation. Applying the interpretation rule (6) for conditionals to example (13) one obtains equation 4 .

$$
\begin{aligned}
(13) & =\operatorname{MUST}_{O, \leq}\left(\operatorname{PRES}_{1}(P), \operatorname{PAST}_{2}(Q)\right) \\
& =\lambda p \cdot \forall p^{\prime}: p^{\prime} \in \operatorname{Min}_{O, \leq}\left(p, \operatorname{PRES}_{1}(P)\right) \rightarrow \operatorname{PAST}_{2}(Q)\left(p^{\prime}\right)
\end{aligned}
$$

Above, we have already calculated the restrictor $\operatorname{Min}_{O, \leq}\left(p, P R E S_{1}(P)\right)$ for a possibility $p=\langle w, t\rangle$ and an antecedent $P R E S_{1} P$ in case $P R E S_{1} P$ is not predetermined at any time $t^{*}$ with $t \leq t^{*}<t_{1}$. This set contains the ontic alternatives $p^{\prime}$ of $p$ whose temporal perspective $t^{\prime}$ is the denotation time $t_{1}$ of the present tense in the antecedent and that make $P$ true at $t_{1}$. At these possibilities now the consequent $P A S T_{2} Q$ is evaluated. Because tenses choose as anchor the local evaluation time the anchor of the past tense $P A S T_{2}$ is set to the temporal perspective $t^{\prime}$ of the possibilities in the restrictor. This time is the denotation time $t_{1}$ of the Simple Present in the antecedent. In consequence the only restriction on $t_{2}$ is that it has to be a time before this anchor time $t^{\prime}=t_{1}$. That means $t_{2}$ can (but does not have to) lie in the future of the utterance time. Hence, we correctly predict the future shift of the anchor time for tenses in the antecedent.

(1) [Let's wait for today's decision regarding his travel arrangements.] Then, If he arrives tomorrow, we'll book his room tonight. 


\subsection{Observation 4}

Observation 4 stated that the shift of the anchor reported in observation 3 is only possible if the denotation time of the tense in the antecedent is indeed a future time. Also this is correctly predicted by the present proposal, because the temporal perspective in the restrictor can only be shifted to the future, if the antecedent indeed refers to the future. We leave the calculation to the reader. ${ }^{14}$

\subsection{Observation 5}

Finally, we observed in section 2 that the future shift of the anchor for tenses in the consequent is not obligatory. Also this is correctly predicted by the present approach. A future shift of the local temporal perspective of the possibilities in the restrictor set only results if (i) the ontic modal base is chosen, and (ii) the eventuality described in the antecedent is not taken to be predetermined at the utterance time. A choice of a different modal base does not automatically lead to future interpretations of tenses on the consequent. For instance, this is not predicted if an epistemic modal base is chosen. Following Kaufmann (2005) we model the doxastic state of some agent as accessibility relation connecting possibilities with identical temporal perspective. ${ }^{15}$ Consequently, the restrictor of the quantifier in the interpretation rule of an epistemic conditional (choosing the doxastic state of the speaker as modal base) can only contain possibilities whose temporal perspective is identical with the utterance time. Thus, tenses in the consequent remain anchored in the utterance time.

As noticed in section 2, Crouch (1993) observes that the cases in which future reference in the antecedent does not lead to a shift of the anchor for the consequent coincide with the cases where the antecedent selects possibilities in which its truth is taken to be predetermined at the utterance time. It should be clear by now that the present approach predicts this to be true in case an ontic modal base is chosen. ${ }^{16}$ But it is also predicted for an epistemic modal base. A statement a speaker makes about the future is about the future in all of his/her epistemic alternatives. The interpretation of the antecedent leads to the selection of all those epistemic alternatives where the future statement is true. Because of the way possibilities are defined, a future statement can only be true in a possibility if it is predetermined at the utterance time. Hence, the interpretation of the antecedent selects possibilities in which the truth of the antecedent is taken to be predetermined.

\footnotetext{
${ }^{14}$ In this connection the reader might want to calculate the predictions made for counterfactual conditionals. The present paper, however, is silent on the semantics of such conditionals.

${ }^{15}$ This an idealization in so far as it implies that the agent knows the actual time. Because of space limitations we cannot go deeper into this issue here.

${ }^{16}$ See footnote 12 .
} 


\section{Discussion}

\subsection{Tenses under modals}

The future shifted interpretations of tenses discussed in this paper for conditional contexts can also be observed for subordinated sentences in the scope of modals (see the examples in (14) borrowed from Crouch (1993)).

(14) a. By 1998 everybody will know someone who died of Aids.

b. Next week, you must show me a problem that you solved on your own.

Because the present approach for the interpretation of tenses in conditional contexts is essentially a modal approach, it should not come as a surprise that it straight forwardly extends to the modal examples. Future shifted anchor times for such subordinated tenses are predicted as long as the modal base of the relevant modality is taken to be the ontic modal base or a modal base that has similar properties concerning the temporal perspective of the involved possibilities. The second sentence, for instance, is based on an deontic modality, for which a similar characteristic of the modal base can be assumed. ${ }^{17}$

\subsection{The upper limit constraint}

In Abusch (1997) an Upper Limit Constraint is proposed to explain the interpretation of tenses in embedded contexts.

Upper limit constraint (ULC)

The local evaluation time is an upper limit for the denotation of tenses.

This constraint is, for instance, employed to explain the observations made in section 3.2.2 for example (10). Given a relative semantics for the tenses that sets their anchor to the local evaluation time, this constraint is not needed for these two examples anymore, but might be necessary to explain the absence of readings in other cases, for instance for double access readings and SOT phenomena that are not discussed in this paper. In Abusch (1997) the ULC is just a postulated constraint. Abusch suggests that it holds because " $\ldots$ future times are not sufficiently determined from the perspective of a now of an intensional context." (Abusch (1997), p.

\footnotetext{
${ }^{17}$ There is an interesting complication pointed out to me by Schwarzchild (p.c.). The sentence (1) containing a deictic temporal adverbial in the subordinated clause is odd.

(1) Next week, you must show me a problem that you solved tomorrow on your own.

So far, the present theory does not make concrete predictions for this sentence, because it does not say anything about the semantics of deictic temporal adverbials. But to account for the oddness of (1) one has to make sure that in case such deictic temporal adverbials occur the local evaluation time has to agree with the deictic centre. It is not per se clear, why this should be the case, nor whether it is generally correct.
} 
24), but she does not propose a explicit derivation of how the constraint emerges. Heim (1994) interprets this constraint as a presupposition of certain syntactic constructions and Abusch (1997) finds this idea appealing. But such a step does not do justice to Abusch (1997)'s basic intuition for how the ULC comes about. The present approach, on the contrary, offers an explanation of the ULC that follows Abusch's ideas. The ULC is a fact that can be proven based on the introduced model theory, in particular the way possibilities and the interpretation of the tenses are defined. This is a pleasing by-product of the present approach.

Fact 1 The denotation of tenses can lie in the future of the local evaluation time, if and only if the eventuality described in the scope of the tense is predetermined at the local evaluation time.

\subsection{Alternative proposals}

Due to space limitations it is not possible to give a comprising account of the existing literature on the issues discussed in this paper. There is, however, one alternative proposal that should be mentioned. Kaufmann (2005) has been the inspiration and starting point of the work presented here. Therefore, it is not surprising that both approaches show a strong similarity. However, the central motivation of the present approach was to overcome some (at least in the eye of the author) less favorable aspects of the approach presented in Kaufmann (2005). This led also to important differences between the proposals.

The central objective of the work presented here was to do without Kaufmann (2005)'s assumption that all English tenses are (implicitly) modalized. This led to the alternative formalization of the asymmetry between past and future and the ontic modal base ${ }^{18}$ defended here. ${ }^{19}$ Another objective of the present work was to avoid Kaufmann (2005)'s assumption that the tenses marked on modals are interpreted in the scope of the modal. This violates the compositional structure of the sentences and leads to empirical difficulties. For instance, one cannot explain why English would and many more past tensed modals in other languages behave as past forms of a modal stem (WOLL for the case of would). Finally, in order to account for the ontic reading of conditionals ${ }^{20}$ Kaufmann (2005) introduces an alternative interpretation rule for ontic conditionals ${ }^{21}$. The special operator occurring in this rule additionally allows him to account to the future shifted anchor of tenses in the consequent of conditionals. In the present approach the difference between the ontic and the epistemic reading of a conditional simply reduces to the difference between the ontic and the epistemic modal base. The temporal properties of these

\footnotetext{
${ }^{18}$ In Kaufmann (2005) this modal base is called the metaphysical modal base.

${ }^{19}$ The present approach is successful in this respect for English, but it might be necessary to introduce an implicit modal to deal with the present tense in German. In contrast to English, the German present tense can also be used if the described eventuality is not predetermined at the utterance time.

${ }^{20}$ Kaufmann (2005) calls this the predictive reading of conditionals.

${ }^{21}$ Kaufmann (2005) proposes that this is not a semantic ambiguity but a pragmatically resolved underspecification of the interpretation rule of conditional sentences.
} 
modal bases are then hold responsible for the different interpretation of the tenses in epistemic and ontic conditionals. No additional difference in the interpretation rule is necessary. ${ }^{22}$

\section{Conclusions}

In this paper a number of observations concerning the interpretation of tense in conditional sentences have been discussed. The paper proposes an explanation of these observations that combines a simple Kratzer-style semantics for conditionals, a non-deictic interpretation of the tenses, as proposed in Abusch (1997), and a non-standard analysis of the ontic modal base. The approach extends to observations concerning the interpretation of tense in the scope of modals and offers an explanation for Abusch (1997)'s Upper Limit Constraint (ULC).

\section{References}

Abusch, D.: 1997, 'Sequence of tense and temporal de re', Linguistics and Philosophy 20, 1-50.

Crouch, R.: 1993, The temporal properties of English conditionals and modals, Doctoral Dissertation, University of Cambridge.

Heim, I.: 1994, 'Comments on Abusch's theory of tense', in H. Kamp (ed.), Ellipsis, tense and questions, 141-170. University of Amsterdam.

Kamp, H.: 1978, 'The logic of historical necessity' . unpublished manuscript.

Kamp, H. and U. Reyle: 1993, From Discourse to Logic. Kluwer, Dordrecht.

Kaufmann, S.: 2005, 'Conditional truth and future reference', Journal of Semantics 22, 231-280.

Kratzer, A.: 1979, 'Conditional necessity and possibility', in R. Bäuerle, U. Egli, and A. von Stechow (eds.), Semantics from different points of view, 387394. Springer, Berlin/Heidelberg/New York.

Kratzer, A.: 1981, 'The notional category of modality', in H.-J. Eikmeyer and H. Rieser (eds.), Words, worlds, and contexts, 387-394. De Gruyter, Berlin/New York.

Quirk, R., S. Greenbaum, G. Leech, and J. Svartvik: 1985, A communicative grammar of the English language, Vol. 2. Longman, London/New York.

Schulz, K.: 2007, Minimal models in semantics and pragmatics: Free choice, exhaustivity, and conditionals, Doctoral Dissertation, University of Amsterdam, Amsterdam.

Stechow, A. von: 2005, 'Semantisches und morphologisches Tempus: Zur temporalen Orientierung von Einstellungen und Modalen', Neue Beiträge zur Germanistischen Linguistik 4.

\footnotetext{
${ }^{22}$ Kaufmann (2005) provides some empirical arguments in favor of his richer semantics. I am not fully convinced by the observations he refers to. More empirical work is needed on this point.
} 